\title{
WEAK REGULARITY OF PROBABILITY MEASURES
}

\author{
DALE SIEGEL \\ Kingsborough Community College \\ Mathematics/Computer Science Department \\ 2001 Oriental Boulevard \\ Brooklyn, New York 11235
}

(Received September 9, 1996 and in revised form January 7, 1997)

\begin{abstract}
This paper examines smoothness attributes of probability measures on lattices which indicate regularity, and then discusses weaker forms of regularity; specifically, weakly regular and vaguely regular. They are obtained from commonly used outer measures, and we study them mainly for the case of $M(\mathcal{L})$ or for those components of $M(\mathcal{L})$ with added smoothness prerequisites. This is a generalization of many concepts presented in my earlier paper (see [1]).
\end{abstract}

KEY WORDS AND PHRASES: Lattice regular, $\sigma$-smooth, and outer measures. Weakly and vaguely regular measures. Normal and complement generated lattices.

\section{AMS SUBJECT CLASSIFICATION CODES: $28 \mathrm{C} 15$.}

\section{INTRODUCTION}

Let $X$ be an arbitrary set and $\mathcal{L}$ a lattice of subsets of $X . A(\mathcal{L})$ denotes the algebra generated by $\mathcal{L}$ and $M(\mathcal{L})$ those finitely additive measures on $A(\mathcal{L}) . M_{\sigma}(\mathcal{L})$ denotes those elements of $M(\mathcal{L})$ that are $\sigma$ smooth on $\mathcal{L}$; while $M_{R}(\mathcal{L})$ denotes those elements of $M(\mathcal{L})$ that are $\mathcal{L}$-regular. To each $\mu \in M(\mathcal{L})$ we will associate a finitely subadditive outer measure $\mu^{\prime}$ on $P(X)$, and to $\mu \in M_{\sigma}(\mathcal{L})$ is associated an outer measure $\mu^{\prime \prime}$. The relationships between $\mu, \mu^{\prime}$, and $\mu^{\prime \prime}$ on $\mathcal{L}$ and $\mathcal{L}^{\prime}$ (the complementary lattice) are investigated. This leads to a consideration of weak notions of regularity, which can be expressed in terms of $\mu^{\prime}$ and $\mu^{\prime \prime}$. In this respect the normal lattices are particularly important since for such lattices regularity of $\mu$ coincides with weak regularity. We show that if $\mu \in N(\mathcal{L})$, those $\mu \in M(\mathcal{L})$ such that for $L_{n} \downarrow L$, $L_{n}, L \in \mathcal{L}, \mu(L)=\inf _{n} \mu\left(L_{n}\right)$ and if $\mathcal{L}$ is complement generated then $\mu$ is weakly regular. Combining these results gives conditions for certain measures to be regular. We adhere to standard lattice and measure terminology which will be used throughout the paper (see e.g. [2-6]) and review some of this in section two for the reader's convenience.

\section{DEFINITIONS AND NOTATIONS}

Let $X$ be an abstract set. Let $\mathcal{L}$ be a lattice of subsets of $X$. We assume throughout that $\emptyset$ and $X$ are in $\mathcal{L}$. If $A \subset X$, then we will denote the complement of $A$ by $A^{\prime}$ (i.e. $A^{\prime}=X-A$ ). If $\mathcal{L}$ is a lattice of subsets of $X$, then $\mathcal{L}^{\prime}=\left\{L^{\prime} \mid L \in \mathcal{L}\right\}$ is the complementary lattice of $\mathcal{L}$.

\section{LATTICE TERMINOLOGY}

DEFINITION 2.1. Let $\mathcal{L}$ be a lattice of subsets of $X$. We say that:

1. $\mathcal{L}$ is a $\delta$-lattice if it is closed under countable intersections; $\delta(\mathcal{L})$ is the lattice of countable intersections of sets of $\mathcal{L}$.

2. $\mathcal{L}$ is disjunctive if and only if $x \in X, L \in \mathcal{L}$, and $x \notin L$ imply there exists $A \in \mathcal{L}$ such that $x \in A$ and $A \cap L=\emptyset$. 
3. $\mathcal{L}$ is complement generated if $L \in \mathcal{L}$ implies $L=\bigcap_{n=1}^{\infty} L_{n}^{\prime}$, where $L_{n} \in \mathcal{L}$.

4. $\mathcal{L}$ is compact if and only if $X=\bigcup_{\alpha} L_{\alpha}^{\prime}, L_{\alpha} \in \mathcal{L}$, implies there exists a finite number of $L_{\alpha}^{\prime}$ that cover $X$.

5. $\mathcal{L}$ is countably compact if and only if $X=\bigcup_{i=1}^{\infty} L_{i}^{\prime}, L_{i} \in \mathcal{L}$, implies there exists a finite number of the $L_{z}^{\prime}$ that cover $X$.

6. $\mathcal{L}$ is countably paracompact if, for every sequence $\left\{L_{n}\right\}$ in $\mathcal{L}$ such that $L_{n} \downarrow \emptyset$, there exists a sequence $\left\{\tilde{L}_{n}\right\}$ in $\mathcal{L}$ such that $L_{n} \subset \tilde{L}_{n}^{\prime}$ and $\tilde{L}_{n}^{\prime} \downarrow \emptyset$.

7. $\mathcal{L}$ is normal if and only if $A, B \in \mathcal{L}$ and $A \cap B=\emptyset$ imply there exists $C, D \in \mathcal{L}$ such that $A \subset C^{\prime}, B \subset D^{\prime}$, and $C^{\prime} \cap D^{\prime}=\emptyset$.

\section{MEASURE TERMINOLOGY}

Let $\mathcal{L}$ be a lattice of subsets of $X . M(\mathcal{L})$ will denote the set of finite-valued, bounded, finitely additive measures on $A(\mathcal{L})$. We may clearly assume throughout that all measures are non-negative.

\section{DEFINITION 2.2.}

1. A measure $\mu \in M(\mathcal{L})$ is said to be $\sigma$-smooth on $\mathcal{L}$ if $L_{n} \in \mathcal{L}$ and $L_{n} \downarrow \emptyset$ imply $\mu\left(L_{n}\right) \rightarrow 0$.

2. A measure $\mu \in M(\mathcal{L})$ is said to be $\sigma$-smooth on $A(\mathcal{L})$ if $A_{n} \in A(\mathcal{L})$ and $A_{n} \downarrow \emptyset$ imply $\mu\left(A_{n}\right) \rightarrow 0$.

3. A measure $\mu \in M(\mathcal{L})$ is said to be $\mathcal{L}$-regular if, for any $A \in A(\mathcal{L})$, $\mu(A)=\sup \{\mu(L): L \subset A, L \in \mathcal{L}\}$.

NOTATION 2.3. If $\mathcal{L}$ is a lattice of subsets of $X$, then we will denote by:

$M_{\sigma}(\mathcal{L})=$ the set of $\sigma$-smooth measures on $\mathcal{L}$ of $M(\mathcal{L})$

$M^{\sigma}(\mathcal{L})=$ the set of $\sigma$-smooth measures on $A(\mathcal{L})$ of $M(\mathcal{L})$

$M_{R}(\mathcal{L})=$ the set of $\mathcal{L}$-regular measures of $M(\mathcal{L})$

$M_{R}^{\sigma}(\mathcal{L})=$ the set of $\mathcal{L}$-regular measures of $M^{\sigma}(\mathcal{L})$

\section{DEFINITION 2.4.}

1. Let $\mu \in M(\mathcal{L})$. Then $\mu \in N(\mathcal{L})$ if $L_{n} \in \mathcal{L}$ and $\bigcap_{n=1}^{\infty} L_{n}=L \in \mathcal{L}$ (in particular, if $\mathcal{L}$ is $\delta$ ), $L_{n} \downarrow$, imply $\mu(L)=\inf \mu\left(L_{n}\right)$.

2. If $\mu \in M(\mathcal{L})$, then the support of $\mu$ is $S(\mu)=\cap\{L \in \mathcal{L} \mid \mu(L)=\mu(X)\}$.

REMARK 2.5. Listed below are a few basic important facts that will be used throughout the paper (see [7,8] for further details):

1. $M_{R}^{\sigma}(\mathcal{L})=M_{R}(\mathcal{L}) \cap M_{\sigma}(\mathcal{L})$

2. $M_{\sigma}(\mathcal{L}) \supset N(\mathcal{L}) \supset M^{\sigma}(L)$

3. If $\mu \in M(\mathcal{L})$, then there exists $\nu \in M_{R}(\mathcal{L})$ such that $\mu \leq \nu(\mathcal{L})$ (i.e. $\mu(L) \leq \nu(L)$, all $L \in \mathcal{L}$ ) and $\mu(X)=\nu(X)$.

\section{REGULAR PROBABILITY MEASURES}

Discussion of $\mathcal{L}$-regular measures $\left(\mu \in M_{R}(\mathcal{L})\right)$ takes place in this section. Conditions for regularity and various resulting properties are examined.

THEOREM 3.1. Let $\mathcal{L}$ be a lattice of subsets of $X$. Then $\mu \in M_{R}(\mathcal{L})$ if and only if $\mu \in M(\mathcal{L})$ and $\mu(A)=\inf \left\{\mu\left(L^{\prime}\right): A \subset L^{\prime}, L \in \mathcal{L}\right\}, A \in A(\mathcal{L})$.

PROOF. 1. Suppose $\mu \in M_{R}(\mathcal{L})$. Then $\mu\left(A^{\prime}\right)=\sup \left\{\mu(L): L \subset A^{\prime}, L \in \mathcal{L}\right\}$. Hence 


$$
\begin{aligned}
\mu(A)=\mu(X)-\mu\left(A^{\prime}\right) & =\mu(X)-\sup \left\{\mu(L): L \subset A^{\prime}, L \in \mathcal{L}\right\} \\
& =\mu(X)-\sup \left\{\mu(L): L^{\prime} \supset A, L \in \mathcal{L}\right\} \\
& =\mu(X)-\sup \left\{\mu(X)-\mu\left(L^{\prime}\right): L^{\prime} \supset A, L \in \mathcal{L}\right\} \\
& =\mu(X)-\mu(X)-\sup \left\{-\mu\left(L^{\prime}\right): L^{\prime} \supset A, L \in \mathcal{L}\right\} .
\end{aligned}
$$

Therefore $\mu(A)=\inf \left\{\mu\left(L^{\prime}\right): A \subset L^{\prime}, L \in \mathcal{L}\right\}$.

2. Reverse of 1 . is sufficient proof.

THEOREM 3.2. Let $\mathcal{L}$ be a lattice of subsets of $X$. Suppose $\mu \in M(\mathcal{L})$ and $\mu\left(L^{\prime}\right)=\sup \left\{\mu(\tilde{L}): \tilde{L} \subset L^{\prime}, \tilde{L} \in \mathcal{L}\right\}$. Then $\mu \in M_{R}(\mathcal{L})$.

PROOF. Suppose $\mu \in M(\mathcal{L})$ and $\mu\left(L^{\prime}\right)=\sup \left\{\mu(\tilde{L}): \tilde{L} \subset L^{\prime}, \tilde{L} \in \mathcal{L}\right\}$. This implies $\mu(L)=\inf \left\{\mu\left(\tilde{L}^{\prime}\right): L \subset \tilde{L}^{\prime}, \tilde{L} \in \mathcal{L}\right\}$, by Theorem 3.1. Let $A \in A(\mathcal{L})$. By definition, $A=\bigcup_{i=1}^{n} L_{i} \cap \widetilde{L}_{i}^{\prime}$, where $L_{i}, \widehat{L}_{\imath} \in \mathcal{L}$ and disjoint. Consider $L \cap \widehat{L} ; L, \widehat{L} \in \mathcal{L}$. Since every $L \in \mathcal{L}$ is $\mathcal{L}^{\prime}$-outer regular with respect to $\mu$, there exists $\tilde{L}^{\prime} \supset L, \tilde{L} \in \mathcal{L}$, such that $\mu(L)+\epsilon>\mu\left(\tilde{L}^{\prime}\right), \epsilon>0$. Then $\tilde{L}^{\prime} \cap \widetilde{L}^{\prime} \supset L \cap \widehat{L}^{\prime}$ and $\tilde{L}^{\prime} \cup \widehat{L}^{\prime} \supset L \cup \widehat{L}^{\prime}$.

$$
\mu\left(L \cap \widetilde{L}^{\prime}\right)=\mu(L)+\mu\left(\widehat{L}^{\prime}\right)-\mu\left(L \cup \widetilde{L}^{\prime}\right) \geq \mu(\widetilde{L})-\epsilon+\mu\left(\widetilde{L}^{\prime}\right)-\mu\left(\tilde{L}^{\prime} \cup \widetilde{L}^{\prime}\right)=\mu\left(\tilde{L}^{\prime} \cap \widetilde{L}^{\prime}\right)-\epsilon .
$$

Consequently, $\mu\left(L \cap \widehat{L}^{\prime}\right)+\epsilon \geq \mu\left(\tilde{L}^{\prime} \cap \widetilde{L}^{\prime}\right)$. Therefore, $L \cap \widetilde{L}^{\prime}$ is $\mathcal{L}^{\prime}$-outer regular with respect to $\mu$. Now, in general, $A=\bigcup_{1}^{n}\left(L_{\imath} \cap \widehat{L}_{i}^{\prime}\right)$, where $L_{i}, \widehat{L}_{i}$ disjoint and $\epsilon>0$. There exists $\tilde{L}_{\imath}^{\prime} \supset L_{\imath} \cap \widehat{L}_{\imath}^{\prime}$ such that $\mu\left(L_{\imath} \cap \widehat{L}_{i}^{\prime}\right)+\frac{\epsilon}{2^{i}}>\mu\left(\tilde{L}_{i}^{\prime}\right)$. Then $\bigcup_{i=1}^{n} \tilde{L}_{i}^{\prime} \supset \bigcup_{1}^{n}\left(L_{i} \cap \widehat{L}_{i}^{\prime}\right)=A$ and $\bigcup_{1}^{n} \tilde{L}_{i}^{\prime} \in \mathcal{L}^{\prime}$.

$$
\mu(A)=\mu\left(\bigcup_{i}^{n}\left(L_{2} \cap \widehat{L}_{i}^{\prime}\right)\right)=\sum_{i=1}^{n} \mu\left(L_{i} \cap \widehat{L}_{i}^{\prime}\right) \geq \sum_{1}^{n} \mu\left(\tilde{L}_{i}^{\prime}\right)-\sum_{1}^{n} \frac{\epsilon}{2^{i}} \geq \mu\left(\bigcup_{1}^{n} \tilde{L}_{i}^{\prime}\right)-\epsilon .
$$

Hence $\mu(A)=\inf \left\{\mu\left(L^{\prime}\right): A \subset L^{\prime}, L \in \mathcal{L}\right\}$. Therefore $\mu \in M_{R}(\mathcal{L})$, by 3.1.

THEOREM 3.3 Let $\mu_{1} \in M_{R}(\mathcal{L}), \mu_{2} \in M(\mathcal{L}), \mu_{1} \leq \mu_{2}(\mathcal{L})$, and $\mu_{1}(X)=\mu_{2}(X)$. Then $\mu_{1}=\mu_{2}$.

PROOF. Suppose $\mu_{1} \leq \mu_{2}(\mathcal{L})$ and let $L \subset \tilde{L}^{\prime}, L, \tilde{L} \in \mathcal{L}$. This implies $\mu_{2} \leq \mu_{1}\left(\mathcal{L}^{\prime}\right)$ and $\mu_{2}(L) \leq \mu_{2}\left(\tilde{L}^{\prime}\right) \leq \mu_{1}\left(\tilde{L}^{\prime}\right)$. If $\mu_{2}(L) \leq \mu_{1}\left(\tilde{L}^{\prime}\right)$, for any $\tilde{L}^{\prime} \supset L$, then $\mu_{2}(L) \leq \inf \left\{\mu_{1}\left(\tilde{L}^{\prime}\right)\right.$ : $\left.L \subset \tilde{L}^{\prime}\right\}=\mu_{1}(L)$, since $\mu \in M_{R}(\mathcal{L})$. Hence $\mu_{2} \leq \mu_{1}(\mathcal{L})$ and, consequently, $\mu_{1}=\mu_{2}(\mathcal{L})$. Therefore $\mu_{1}=\mu_{2}$ since $\mu_{1}(X)=\mu_{2}(X)$.

THEOREM 3.4. Let $\mathcal{L}$ be a lattice of subsets of $X$. Suppose $\mu \in M_{R}(\mathcal{L})$ and $\mu \in M_{\sigma}(\mathcal{L})$. Then $\mu \in M^{\sigma}(\mathcal{L})$.

PROOF. Given $\mu \in M_{R}(\mathcal{L})$ and $\mu \in M_{\sigma}(\mathcal{L})$. Let $\left\{A_{n}\right\}$ be in $A(\mathcal{L})$ and $A_{n} \downarrow \emptyset$. Then there exists $L_{n} \subset A_{n}, L_{n} \in \mathcal{L}$, and $\mu\left(A_{n}\right)-\frac{\epsilon}{2^{n}}<\mu\left(L_{n}\right)$, since $\mu \in M_{R}(\mathcal{L})$. Now, $L_{1}, L_{1} \cap L_{2}, L_{1} \cap L_{2} \cap L_{3}, \ldots$ are in $\mathcal{L}$ and $1 \emptyset$. So $\mu\left(L_{1} \cap L_{2}\right) \leq \mu\left(A_{1} \cap A_{2}\right)=\mu\left(A_{2}\right) \leq \mu\left(L_{1} \cap L_{2}\right)+\frac{\epsilon}{2}+\frac{\epsilon}{4}$. By induction, $\mu\left(\bigcap_{i=1}^{n} A_{i}\right) \leq \mu\left(\bigcap_{1}^{n} L_{i}\right)+\sum_{i=1}^{n} \frac{\epsilon}{2^{i}}$ for all $n$. Consequently, we may assume $L_{n} \downarrow \emptyset$ and $\mu\left(A_{n}\right)<\mu\left(L_{n}\right)+\epsilon$, all $n$. Then $\lim _{n \rightarrow \infty} \mu\left(A_{n}\right) \leq \lim _{n \rightarrow \infty} \mu\left(L_{n}\right)+\epsilon$; and $\lim _{n \rightarrow \infty} \mu\left(L_{n}\right)=0$ since $\mu \in M_{\sigma}(\mathcal{L})$. This implies $\lim _{n} \mu\left(A_{n}\right) \leq \epsilon$ and $\epsilon>0$. Hence $\lim _{n} \mu\left(A_{n}\right)=0$. Therefore $\mu \in M^{\sigma}(\mathcal{L})$, since $\mu$ is countably additive on $A(\mathcal{L})$.

THEOREM 3.5. Let $\mu \leq \nu(\mathcal{L})$, where $\mu \in M(\mathcal{L}), \nu \in M_{R}(\mathcal{L})$, and $\mu(X)=\nu(X)$. If $\mathcal{L}$ is normal, then $\nu\left(L^{\prime}\right)=\sup \left\{\mu(\tilde{L}): \tilde{L} \subset L^{\prime} ; L, \tilde{L} \in \mathcal{L}\right\}$.

PROOF. Since $\nu \in M_{R}(\mathcal{L}), \nu\left(L^{\prime}\right)=\sup \{\nu(\tilde{L}): \tilde{L} \subset L ; L, \tilde{L} \in \mathcal{L}\}$. This implies $\nu\left(L^{\prime}\right)-$ $\epsilon<\nu(\tilde{L}), \epsilon>0$, for some $\tilde{L} \in \mathcal{L}$ where $\tilde{L} \subset L$. By normality, $\tilde{L} \subset A^{\prime} \subset B \subset L^{\prime}$, where $A, B \in \mathcal{L}$. 
Then $\nu\left(L^{\prime}\right)-\epsilon<\nu(\tilde{L}) \leq \nu\left(A^{\prime}\right) \leq \mu\left(A^{\prime}\right) \leq \mu(B) \leq \nu(B) \leq \nu(L)$. Hence $\nu\left(L^{\prime}\right)=\sup \{\mu(B)$ : $\left.B \subset L^{\prime}, B \in \mathcal{L}\right\}$, and $\mu(B) \sim \mu(\tilde{L})$ by an $\epsilon$-argument. Therefore $\nu\left(L^{\prime}\right)=\sup \left\{\mu(\tilde{L}): \tilde{L} \subset L^{\prime}, \tilde{L} \in \mathcal{L}\right\}$.

THEOREM 3.6. Suppose $\mu \in M_{R}(\mathcal{L})$ and $\lambda \in M_{R}\left(\mathcal{L}^{\prime}\right)$ such that $\mu \leq \lambda\left(\mathcal{L}^{\prime}\right)$. Then $\mathcal{L}$ is normal if and only if $\mu\left(L^{\prime}\right)=\sup \left\{\lambda(A): A \subset L^{\prime}, A \in \mathcal{L}\right\}$.

\section{PROOF.}

1. $\mu \leq \lambda\left(\mathcal{L}^{\prime}\right)$ implies $\lambda \leq \mu(\mathcal{L})$, by regularity. Therefore, if $\mathcal{L}$ is normal, then $\mu\left(L^{\prime}\right)=\sup \{\lambda(A)$ : $\left.A \subset L^{\prime}, A \in \mathcal{L}\right\}$ by 3.5 .

2. Suppose $\mu\left(L^{\prime}\right)=\sup \left\{\lambda(A): A \subset L^{\prime}, A \in \mathcal{L}\right\}$. Let $\mu_{1}, \mu_{2} \in M_{R}(\mathcal{L})$ such that $\mu \leq \mu_{1}(\mathcal{L})$ and $\mu \leq \mu_{2}(\mathcal{L})$. Then $\mu_{1} \leq \mu \leq \lambda\left(\mathcal{L}^{\prime}\right)$ and $\mu_{2} \leq \mu \leq \lambda\left(\mathcal{L}^{\prime}\right)$. This implies $\mu_{1}\left(L^{\prime}\right)=\mu_{2}\left(L^{\prime}\right)=$ $\sup \left\{\lambda(A): A \subset L^{\prime}, A \in \mathcal{L}\right\}$. Hence $\mu_{1}=\mu_{2}$. Therefore, $\mathcal{L}$ is normal.

THEOREM 3.7. Suppose $\mathcal{L}$ is normal and complement generated. Then $\mu \in N(\mathcal{L})$ implies $\mu \in M_{R}^{\sigma}(\mathcal{L})$.

PROOF. Since $\mathcal{L}$ is complement generated, there exists $L, L_{n} \in \mathcal{L}$ such that $L=\bigcap_{n=1}^{\infty} L_{n}^{\prime}$, where $L_{n} \downarrow$. By normality, $L \subset A_{n}^{\prime} \subset B_{n} \subset L_{n}^{\prime}$, where $A_{n}, B_{n} \in \mathcal{L}$, and we may assume that $A_{n} \downarrow, B_{n} \downarrow$. Then $L=\bigcap_{n} B_{n}=\bigcap_{n} L_{n}^{\prime}$. Now let $\mu \in N(\mathcal{L})$. This implies $\mu(L)=\inf _{n} \mu\left(B_{n}\right)=\inf _{n} \mu\left(A_{n}^{\prime}\right)$. Hence $\mu \in M_{R}(\mathcal{L})$ by 3.1 , and $N(\mathcal{L}) \subset M_{\sigma}(\mathcal{L})$ by 2.5 . Therefore $\mu \in M_{R}^{\sigma}(\mathcal{L})$.

\section{OUTER MEASURES}

In this section we consider $\mu \in M(\mathcal{L})$, and associate with it certain "outer measures" $\mu^{\prime}$ and $\mu^{\prime \prime}$. In general, they differ from the customary induced "outer measures" $\mu^{*}$ and $\mu^{*}$. We seek to investigate the interplay of these outer measures on the lattice $\mathcal{L}$ and, conversely, the effect of $\mathcal{L}$ on them.

DEFINITION 4.1. Let $\mu \in M(\mathcal{L})$ such that $\mu \geq 0$ and let $E$ be a subset of $X$.

1. $\mu^{\prime}(E)=\inf \left\{\mu\left(L^{\prime}\right): E \subset L^{\prime}, L \in \mathcal{L}\right\}$ is a finitely-subadditive outer measure.

2. $\mu^{\prime \prime}(E)=\inf \left\{\sum_{n=1}^{\infty} \mu\left(L_{n}^{\prime}\right): E \subset \bigcup_{n=1}^{\infty} L_{n}^{\prime}, L_{n} \in \mathcal{L}\right\}$ is a countably-subadditive outer measure.

3. $\mu^{\bullet}(E)=\inf \{\mu(A): E \subset A, A \in A(\mathcal{L})\}$ is a finitely-subadditive outer measure.

4. $\mu^{*}(E)=\inf \left\{\sum_{i=1}^{\infty} \mu\left(A_{i}\right): E \subset \bigcup_{i=1}^{\infty} A_{\imath}, A_{i} \in A(\mathcal{L})\right\}$ is a countably-subadditive outer measure.

\section{DEFINITION 4.2.}

1. Suppose $\nu$ is an outer measure and let $E$ be a subset of $X$. Then $E \in \mathcal{S}_{\nu}$, the set of $\nu$-measurable sets, if $\nu(A)=\nu(A \cap E)+\nu\left(A \cap E^{\prime}\right)$ for all $A \subset X$.

2. $\nu$ is said to be a regular outer measure if, for $A, E \subset X$, there exists $E \in \mathcal{S}_{\nu}$ such that $A \subset E$ and $\nu(A)=\nu(E)$.

PROPERTY 4.3. Proofs will be omitted.

1. If $\mathcal{L}$ is countably compact and $\mu \in M(\mathcal{L})$, then $\mu^{\prime}=\mu^{\prime \prime}(\mathcal{L})$.

2. If $\mu \in N(\mathcal{L})$, then $\mu^{\prime}=\mu^{\prime \prime}\left(\mathcal{L}^{\prime}\right)$.

3. $\mu \in M_{\sigma}(\mathcal{L})$ and $\mu^{\prime}=\mu^{\prime \prime}\left(\mathcal{L}^{\prime}\right)$, where $\mu^{\prime \prime}$ is regular, imply $\mu \in N(\mathcal{L})$.

4. If $\mu \in N(\mathcal{L})$ and $\mathcal{L}$ is $\delta$, then $\mu^{\prime}=\mu^{\prime \prime}(\mathcal{L})$.

5. Suppose $\mu \in N(\mathcal{L}), \mathcal{L}$ is $\delta$, and $\mathcal{L} \subset \mathcal{S}_{\mu^{\prime \prime}}$. Then $\mu \in M_{R}^{\sigma}(\mathcal{L})$.

THEOREM 4.4. Let $\mu \in M_{\sigma}(\mathcal{L})$. Then

(a) $\mu(X)=\mu^{\prime \prime}(X)$,

(b) $\mu \leq \mu^{\prime \prime} \leq \mu^{\prime}(\mathcal{L})$,

(c) $\mu^{\prime \prime} \leq \mu=\mu^{\prime}\left(\mathcal{L}^{\prime}\right)$.

PROOF. (a) Clearly $\mu^{\prime \prime}(X) \leq \mu(X)$. If $\mu^{\prime \prime}(X)<\mu(X)$, then there exists $L_{i}^{\prime} \in \mathcal{L}^{\prime}, i=1,2, \ldots$, such that $X=\bigcup_{i=1}^{\infty} L_{i}^{\prime}$ and $\sum_{i=1}^{\infty} \mu\left(L_{i}^{\prime}\right)<\mu(X)$. But $\sum_{i=1}^{\infty} \mu\left(L_{i}^{\prime}\right)=\lim _{n \rightarrow \infty} \sum_{1}^{n} \mu\left(L_{i}^{\prime}\right) \geq \lim _{n \rightarrow \infty} \mu\left(\bigcup_{1}^{n} L_{i}^{\prime}\right)$. Also 
$\bigcup_{1}^{n} L_{\imath}^{\prime} \uparrow X$ and $\bigcup_{1}^{n} L_{i}^{\prime} \in \mathcal{L}^{\prime}$. This implies that $\lim _{n} \mu\left(\bigcup_{1}^{n} L_{\imath}^{\prime}\right)=\mu(X)$ since $\mu \in M_{\sigma}(\mathcal{L})$. Therefore $\mu(X)=\mu^{\prime \prime}(X)$.

(b) Suppose there exists $L \in \mathcal{L}$ such that $\mu(L)>\mu^{\prime \prime}(L)$. Then $\mu^{\prime \prime}(X) \leq \mu^{\prime \prime}(L)=$ $\mu^{\prime \prime}\left(L^{\prime}\right)<\mu(L)+\mu^{\prime \prime}\left(L^{\prime}\right)$. Then $\mu^{\prime \prime}(X) \leq \mu^{\prime \prime}(L)+\mu^{\prime \prime}\left(L^{\prime}\right)<\mu(L)+\mu^{\prime \prime}\left(L^{\prime}\right)$, but $\mu^{\prime \prime} \leq \mu(\mathcal{L})$. This implies $\mu^{\prime \prime}(X)<\mu(L)+\mu\left(L^{\prime}\right)=\mu(X)$, which contradicts (a). Hence $\mu \leq \mu^{\prime \prime}(\mathcal{L})$; and $\mu^{\prime \prime} \leq \mu^{\prime}$ everywhere clearly. Thus $\mu^{\prime \prime} \leq \mu^{\prime}(\mathcal{L})$. Therefore $\mu \leq \mu^{\prime \prime} \leq \mu^{\prime}(\mathcal{L})$.

(c) Clearly $\mu^{\prime \prime} \leq \mu^{\prime}\left(\mathcal{L}^{\prime}\right)$ and, by definition, $\mu=\mu^{\prime}\left(\mathcal{L}^{\prime}\right)$. Therefore $\mu^{\prime \prime} \leq \mu=\mu^{\prime}\left(\mathcal{L}^{\prime}\right)$.

THEOREM 4.5. Suppose $\nu$ is a finite, regular, finitely-subadditive outer measure defined on $P(X)$, the set of all subsets of $X$. Then $E \in \mathcal{S}_{\nu}$ if and only if $\nu(X)=\nu(E)+\nu\left(E^{\prime}\right)$.

PROOF. 1. Suppose $\nu$ is a finitely-subadditive regular outer measure and $E \in \mathcal{S}_{\nu}$. Then $\nu(E)+\nu\left(E^{\prime}\right)=\nu(X)$, clearly.

2. Suppose $\nu$ is a finitely-subadditive regular outer measure and $\nu(X)=\nu(E)+\nu\left(E^{\prime}\right)$. Let $B \in \mathcal{S}_{\nu}$. Then, by regularity, there exists a set $F \subset X$ such that $F \subset B$ and $\nu(F)=\nu(B)$. Then, since $B \in \mathcal{S}_{\nu}, \nu(E)=\nu(E \cap B)+\nu\left(E \cap B^{\prime}\right)$ and $\nu\left(E^{\prime}\right)=\nu\left(E^{\prime} \cap B\right)+\nu\left(E^{\prime} \cap B^{\prime}\right)$. So

$$
\begin{aligned}
\nu(X)=\nu(E)+\nu\left(E^{\prime}\right)= & \nu(E \cap B)+\nu\left(E \cap B^{\prime}\right)+\nu\left(E^{\prime} \cap B\right)+\nu\left(E^{\prime} \cap B^{\prime}\right) \\
& \geq \nu(B)+\nu\left(B^{\prime}\right)=\nu(X),
\end{aligned}
$$

' since $B \in \mathcal{S}_{\nu}$. Also, $\nu(B \cap E)+\nu\left(B \cap E^{\prime}\right)+\nu\left(B^{\prime} \cap E\right)+\nu\left(B^{\prime} \cap E^{\prime}\right)=\nu(B)+\nu\left(B^{\prime}\right)$ since all $\nu(X)=$ finite measure. Now subtract from the equation above $\nu\left(B^{\prime} \cap E\right)+\nu\left(B^{\prime} \cap E^{\prime}\right) \geq \nu\left(B^{\prime}\right)$, which is true by the finite subadditivity of $\nu$. Then $\nu(B \cap E)+\nu\left(B \cap E^{\prime}\right) \leq \nu(B)$. Also, $F \cap E \subset B \cap E$ and $F \cap E^{\prime} \subset B \cap E^{\prime}$. This implies

$$
\nu(F \cap E)+\nu\left(F \cap E^{\prime}\right) \leq \nu(B \cap E)+\nu\left(B \cap E^{\prime}\right) \leq \nu(B)=\nu(F) .
$$

Hence $\nu(F)=\nu(F \cap E)+\nu\left(F \cap E^{\prime}\right)$. Therefore $E \in \mathcal{S}_{\nu}$.

THEOREM 4.6. Suppose $\mu \leq \nu(\mathcal{L})$, where $\mu \in M(\mathcal{L})$ and $\nu \in M_{R}(\mathcal{L})$. Then:

(a) $\mu \leq \nu=\nu^{\prime} \leq \mu^{\prime}(\mathcal{L})$

(b) if $\mathcal{L}$ is normal, then $\mu^{\prime}=\nu^{\prime}(\mathcal{L})$.

PROOF. (a) Since $\nu \in M_{R}(\mathcal{L}), \nu(E)=\nu^{\prime}(E)=\inf \left\{\nu\left(L^{\prime}\right): E \subset L^{\prime}, L \in \mathcal{L}\right\}$. Also, $\mu \leq \nu(\mathcal{L})$ implies $\nu \leq \mu\left(\mathcal{L}^{\prime}\right)$, which implies $\nu^{\prime} \leq \mu^{\prime}(\mathcal{L})$ and $\nu^{\prime} \leq \mu^{\prime}\left(\mathcal{L}^{\prime}\right)$. Therefore $\mu \leq \nu=\nu^{\prime} \leq \mu^{\prime}(\mathcal{L})$.

(b) Let $L \in \mathcal{L}$. Then, by normality,

$$
\begin{aligned}
\nu^{\prime}(L)=\nu(L)=\nu(X)-\nu\left(L^{\prime}\right) & =\nu(X)-\sup \left\{\nu(\tilde{L}): \tilde{L} \subset L^{\prime}, \tilde{L} \in \mathcal{L}\right\} \\
& =\nu(X)-\sup \left\{\mu(\tilde{L}): \tilde{L} \subset L^{\prime}, \tilde{L} \in \mathcal{L}\right\} \\
& =\inf \left\{\mu\left(\tilde{L}^{\prime}\right): \tilde{L}^{\prime} \supset L\right\}=\mu^{\prime}(L) .
\end{aligned}
$$

Therefore $\mu^{\prime}=\nu^{\prime}(\mathcal{L})$.

\section{WEAKER NOTIONS OF REGULARITY}

Previously we have considered some properties related to $\mu \in M_{R}(\mathcal{L})$. We now want to consider weaker notions of regularity, and see when they might coincide with regularity; and, in general, to investigate their properties and interplay with the underlying lattice.

DEFINITION 5.1. Let $L \in \mathcal{L}$, where $\mathcal{L}$ is a lattice of subsets of $X$.

1. A measure $\mu \in M(\mathcal{L})$ is said to be weakly regular if $\mu\left(L^{\prime}\right)=\sup \left\{\mu^{\prime}(\tilde{L}): \tilde{L} \subset L^{\prime}, \tilde{L} \in \mathcal{L}\right\}$.

2. A measure $\mu \in M_{\sigma}(\mathcal{L})$ is said to be vaguely regular if $\mu\left(L^{\prime}\right)=\sup \left\{\mu^{\prime \prime}(\tilde{L}): \tilde{L} \subset L^{\prime}, \tilde{L} \in \mathcal{L}\right\}$.

NOTATION 5.2.

$M_{W}(\mathcal{L})=$ the set of weakly regular measures of $M(\mathcal{L})$

$M_{V}(\mathcal{L})=$ the set of vaguely regular measures of $M_{\sigma}(\mathcal{L})$

LEMMA 5.3. $M_{R}^{\sigma}(\mathcal{L}) \subset M_{V}(\mathcal{L}) \subset M_{W}(\mathcal{L}) \cap M_{\sigma}(\mathcal{L})$ 
REMARK 5.4. If $\mu^{\prime}=\mu^{\prime \prime}(\mathcal{L})$, then $M_{V}(\mathcal{L})=M_{W}(\mathcal{L}) \cap M_{\sigma}(\mathcal{L})$. This occurs if:

(a) $\mathcal{L}$ is countably compact,

(b) $\mu \in N(\mathcal{L})$ and $\mathcal{L}$ is $\delta$,

(c) $\mathcal{L}$ is normal and complement generated,

(d) $\mathcal{L}$ is $\delta$-normal.

THEOREM 5.5. Suppose $\mu \leq \nu(\mathcal{L})$, where $\mu \in M_{W}(\mathcal{L})$ and $\nu \in M_{R}(\mathcal{L})$. Then $\mu^{\prime}=\nu(\mathcal{L})$ implies $\mu=\nu$.

PROOF. Let $M_{W}(\mathcal{L}) \ni \mu \leq \nu \in M_{R}(\mathcal{L})$ and suppose $\mu^{\prime}=\nu(\mathcal{L})$. Then $\mu \leq \nu=\nu^{\prime} \leq \mu^{\prime}(\mathcal{L})$ by 4.6. Now, $\mu \in M_{W}(\mathcal{L})$ implies $\mu\left(L^{\prime}\right)=\sup \left\{\mu^{\prime}(\tilde{L}): \tilde{L} \subset L^{\prime} ; L, \tilde{L} \in \mathcal{L}\right\}$ and $\nu \in M_{R}(\mathcal{L})$ implies $\nu\left(L^{\prime}\right)=\sup \left\{\nu(\tilde{L}): \tilde{L} \subset L^{\prime}, L, \tilde{L} \in \mathcal{L}\right\} . \quad$ Then, since $\mu^{\prime}=\nu(\mathcal{L}), \mu\left(L^{\prime}\right)=\nu\left(L^{\prime}\right)$, which implies $\mu=\nu\left(\mathcal{L}^{\prime}\right)$. Therefore $\mu=\nu$, since $\mu(X)=\nu(X)$.

THEOREM 5.6. Suppose $\mathcal{L}$ is complement generated. If $\mu \in N(\mathcal{L})$ and $\mu^{\prime \prime}$ is a regular outermeasure, then $\mu \in M_{V}(\mathcal{L}) \subset M_{W}(\mathcal{L}) \cap M_{\sigma}(\mathcal{L})$.

PROOF. Suppose $\mathcal{L}$ is complement generated and $\mu \in N(\mathcal{L})$. Then $\mu \in M_{\sigma}(\mathcal{L})$ by 2.5 ; and $\mu=\mu^{\prime}=\mu^{\prime \prime}\left(\mathcal{L}^{\prime}\right)$, by 4.3 and 4.4. Now let $L \in \mathcal{L}$. Then, since $\mathcal{L}$ is complement generated, $L=\bigcap_{n=1}^{\infty} L_{n}^{\prime}$, $L_{n} \in \mathcal{L}, L_{n}^{\prime} \downarrow$. By the regularity of $\mu^{\prime \prime}$ and the fact that $L^{\prime} \subset \bigcap_{n=1}^{\infty} L_{n}^{\prime}$, we have $\mu^{\prime \prime}\left(L^{\prime}\right)=\lim _{n} \mu^{\prime \prime}\left(L_{n}\right)$. But $\mu=\mu^{\prime}=\mu^{\prime \prime}\left(\mathcal{L}^{\prime}\right)$ since $\mu \in N(\mathcal{L})$. Thus $\mu\left(L^{\prime}\right)=\sup \left\{\mu^{\prime \prime}(\tilde{L}): \tilde{L} \subset L^{\prime}, \tilde{L} \in \mathcal{L}\right\}$. Hence $\mu \in M_{V}(\mathcal{L})$. Therefore, by $5.3, \mu \in M_{V}(\mathcal{L}) \subset M_{W}(\mathcal{L}) \cap M_{\sigma}(\mathcal{L})$.

THEOREM 5.7. Suppose $\mathcal{L}$ is normal and $\mu \in M_{W}(\mathcal{L})$. Then $\mu \in M_{R}(\mathcal{L})$.

PROOF. Suppose $\mathcal{L}$ is normal and $\mu \in M_{W}(\mathcal{L})$. Let $\mu \leq \nu(\mathcal{L})$, where $\nu \in M_{R}(\mathcal{L})$. Then, using 4.6,

$$
\begin{aligned}
\nu\left(L^{\prime}\right) & =\sup \left\{\nu(\tilde{L}): \tilde{L} \subset L^{\prime} ; L, \tilde{L} \in \mathcal{L}\right\}=\sup \left\{\nu^{\prime}(\tilde{L}): \tilde{L} \subset L^{\prime} ; L, \tilde{L} \in \mathcal{L}\right\} \\
& =\sup \left\{\mu^{\prime}(\tilde{L}): \tilde{L} \subset L^{\prime} ; L, \tilde{L} \in \mathcal{L}\right\}=\mu\left(L^{\prime}\right) \text { since } \mu \in M_{W}(\mathcal{L}) .
\end{aligned}
$$

So $\mu=\nu\left(\mathcal{L}^{\prime}\right)$, which implies $\mu=\nu$ since $\mu(X)=\nu(X)$. Therefore $\mu \in M_{R}(\mathcal{L})$.

REMARK 5.8. We saw in Theorem 5.7 that if $\mathcal{L}$ is normal, then $M_{W}(\mathcal{L})=M_{R}(\mathcal{L})$. However, the converse is not true. For example, let $\mathcal{L}=\{\emptyset, X, A, B, A, \cup B\}$, where $A, B \subset X(A, B \neq \emptyset)$ such that $A \cap B=\emptyset$ and $A \cup B \neq X$. Here $\mathcal{L}$ is clearly not normal, but $M_{W}(\mathcal{L})=M_{R}(\mathcal{L})$.

\section{REFERENCES}

[1] SIEGEL, D., Outer measures and weak regularity of measures, Internat. Jour. Math. \& Math. Sci. 18, no. 1 (1995), 49-58.

[2] ALEXANDROFF, A.D., Additive set-functions in abstract spaces (Chapter I), Mat. Sb. 8 (1940), 307-348.

[3] ALEXANDROFF, A.D., Additive set-functions in abstract spaces (Chapters II-III), Mat. Sb. 9 (1941), 563-628.

[4] ALEXANDROFF, A.D., Additive set-functions in abstract spaces (Chapters IV-VI), Mat. Sb. 13 (1943), 169-238.

[5] FROLIK, Z., Prime filters with the C.I.P., Comm. Math. Univ. Carolinae 13 (1972), 553-575.

[6] SZETO, M., Measure repleteness and mapping preservations, Jour. of the Indian Math. Soc. 43 (1979), 35-52.

[7] CAMACHO, JR., J., On maximal measures with respect to a lattice, Internat. Jour. Math. \& Math. Sci. 14, no. 1 (1991), 93-98.

[8] EID, G., On maximal measures and Wallman spaces, Internat. Jour. Math. \& Math. Sci. 13, no. 1 (1990), 31-38. 


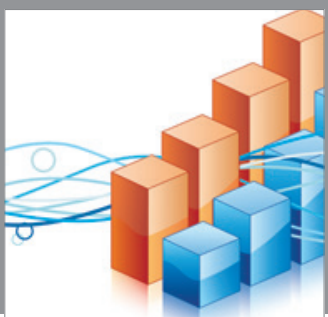

Advances in

Operations Research

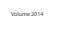

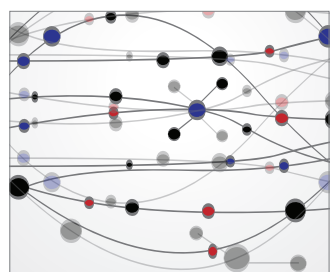

\section{The Scientific} World Journal
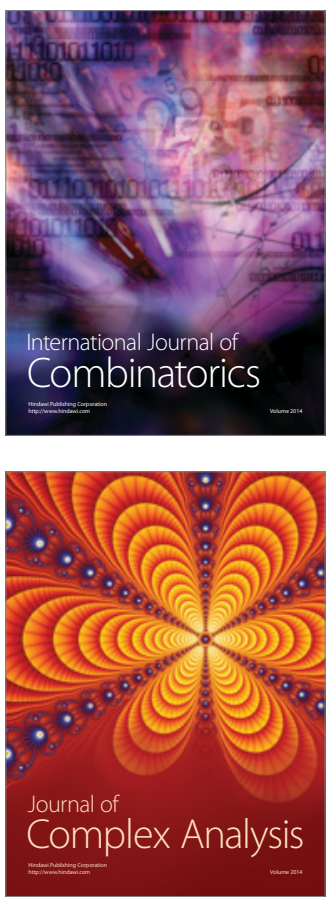

International Journal of

Mathematics and

Mathematical

Sciences
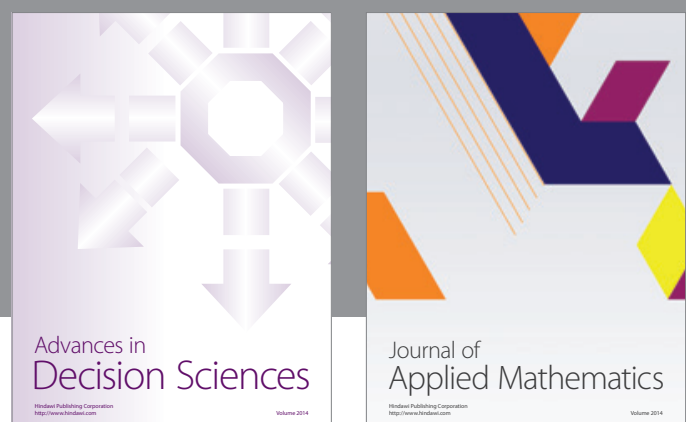

Journal of

Applied Mathematics
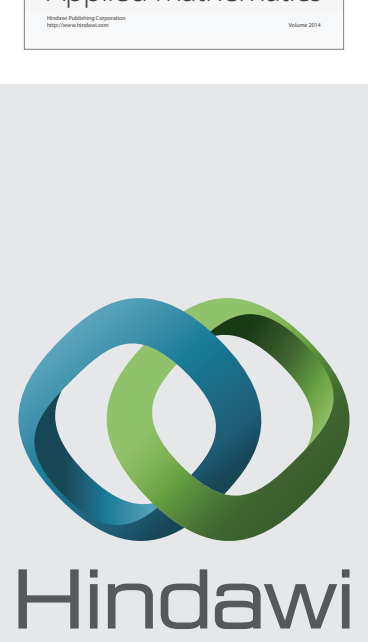

Submit your manuscripts at http://www.hindawi.com
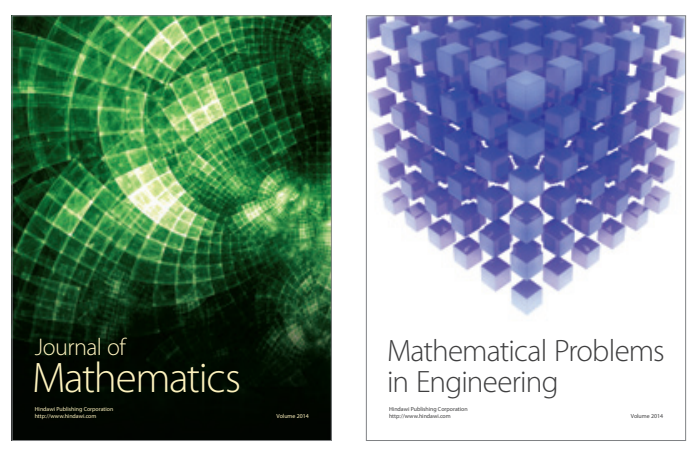

Mathematical Problems in Engineering
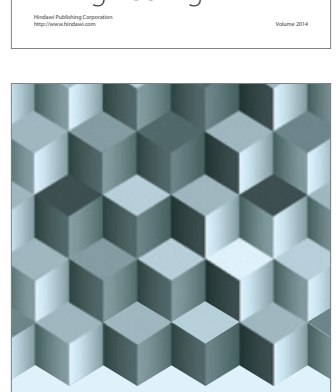

Journal of

Function Spaces
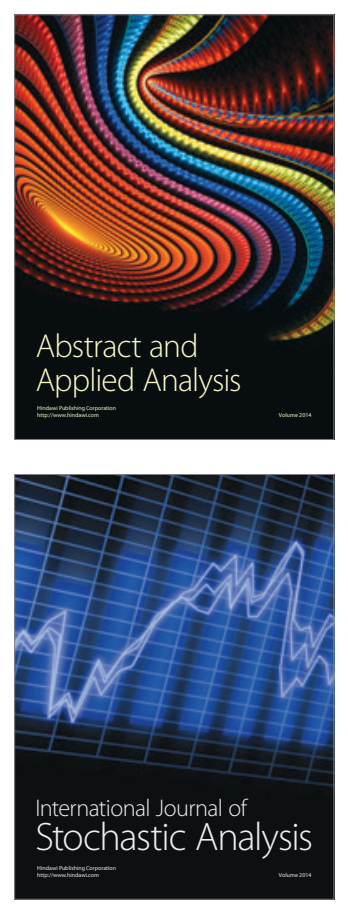

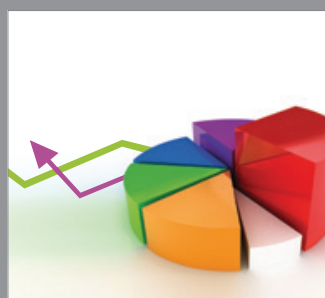

ournal of

Probability and Statistics

Promensencen
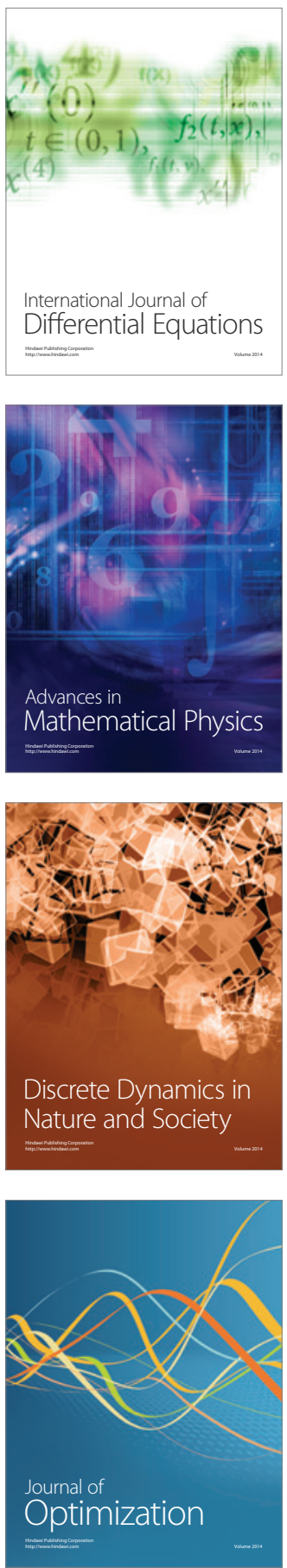